Revista científica, arbitrada e indizada, bajo la modalidad electrónica.

Recibido: $12 / 02 / 2020$

Aceptado: 18/02/2020

\title{
PENSAMIENTO POSITIVO: UNA APROXIMACIÓN CUALITATIVA A LA GERENCIA DE VIDA LABORAL EN ORGANISMOS PÚBLICOS VENEZOLANOS
}

Positive thinking: a qualitative approach to the labor lite management in Venezuelan public organizations

\author{
Carlos Daniel Henríquez Jiménez ${ }^{1}$ \\ carlosdhenriquez@gmail.com \\ Ramiro Aurelio Buitrago Acuña ${ }^{2}$ \\ dr.buitrago86@gmail.com \\ Venezuela
}

\section{RESUMEN}

El manuscrito tiene como propósito, instruir que el pensamiento positivo, favorece las relaciones interpersonales y la capacidad innovadora en el contexto organizacional, hacia una gerencia de vida laboral exitosa. Se trata de una investigación apoyada en el paradigma cualitativo, aplicándose el método etnográfico, con enfoque introspectivo-vivencial, donde el registro de grabación, observación participante, documentación y la entrevista semiestructurada, fueron las técnicas aprovechadas, con sustento en el registro informativo por computadora, la grabadora digital, el diario de campo y la matriz de análisis de la categoría, como instrumentos de investigación aplicados. Con una muestra de sesenta y tres (63) sujetos, reducida intencionalmente a un muestreo de tres (03) sujetos, trabajadores de las unidades seleccionadas para tal fin. Tras el análisis y discusión de los hallazgos alcanzados, se planteó la posibilidad de marcar un rumbo distinto en el sector Público Venezolano, bajo la égida de flexibilidad, dinamismo, emprendimiento e innovación, forjando transformaciones orgánicas, ajustadas a la realidad contemporánea. Concluyéndose que los funcionarios públicos que participaron como sujetos de estudio, cuentan con diferentes cualidades que robustecen el ejercicio de la función pública, al igual

\footnotetext{
${ }^{1}$ Participante con opción a grado en el programa de estudios doctorales en Ciencias Gerenciales / Magister Scientiarum en Derecho Mercantil egresado de la Universidad Privada Dr. Rafael Belloso Chacín (URBE), Abogado egresado de La Universidad del Zulia (LUZ)/ Fiscal Provisorio en la Fiscalía Vigésimo Tercera del Ministerio Público de la Circunscripción Judicial del Estado Zulia con competencia en materia de drogas/ Investigador Independiente. Orcid; http://orcid.org/0000-0002-8668-7768.

${ }^{2}$ Doctor en Ciencias Gerenciales/ Magister Scientiarum en Gerencia de Recursos Humanos/ Diplomado en Ciencias Forenses y Criminalísticas/ Diplomado en el Procedimiento Penal Venezolano egresado de la Universidad Privada Dr. Rafael Belloso Chacín (URBE), Abogado egresado de La Universidad del Zulia (LUZ), Abogado IV para la Alcaldía Bolivariana del Municipio Miranda - Edo. Zulia - Venezuela/ Investigador Independiente. Enlace ORCID: http://orcid.org/0000-0002-8563-7662.
} 


\title{
Revista científica, arbitrada e indizada, bajo la modalidad electrónica.
}

algunas falencias de tipo técnico que pueden ser corregidas con apoyo a los estamentos internos.

Palabras clave: Pensamiento positivo, gerencia de vida laboral y sector público.

\begin{abstract}
The purpose of the manuscript is to instruct that positive thinking favors interpersonal relationships and innovative capacity in the organizational context, towards successful work life management. It is an investigation supported by the qualitative paradigm, where the ethnographic method was applied, with an introspective-experiential approach, where the recording of recording, participant observation, documentation and the semi-structured interview were the techniques used, based on the informative record. by computer, the digital recorder, the field diary and the analysis matrix of the category, as applied research instruments. With a sample of sixty-three (63) subjects, intentionally reduced to a sample of three (03) subjects, workers from the analysis units selected for this purpose. After the analysis and discussion of the findings, the possibility of setting a different course in the Venezuelan Public sector was raised, under the aegis of flexibility, dynamism, entrepreneurship and innovation, forging organic transformations, adjusted to contemporary reality. Concluding that the public officials, who participated as study subjects, have different qualities that strengthen the exercise of the public function and some technical shortcomings that can be corrected with support to the internal levels.
\end{abstract}

Key words: Positive thinking, work life management and public sector.

\section{INTRODUCCIÓN}

Se considera al pensamiento positivo como filosofía de vida, hacia una existencia mucho más fácil y feliz, al advertir su importancia capital, pues de acuerdo a como piense el individuo, así tiende a sentirse y comportarse; cuando una persona interactúa con otras de forma tanto segura como decidida, es porque se siente de tal manera, esta percepción de seguridad, surge con ocasión a lo que ha pensado de sí misma, en este sentido, esta matriz de ideas da origen a sentimientos que le llevan a un comportamiento seguro, afirmativo y decidido. Según Amaya (2020), sucede que:

Las organizaciones requieren de un clima organizacional armónico que les permita a los trabajadores mantener un ambiente estable de trabajo, donde las relaciones personales y la comunicación eficaz fluyan, con la finalidad de que los mensajes y la información transferida puedan 


\section{Revista científica, arbitrada e indizada, bajo la modalidad electrónica.}

tener el significado apropiado para la toma de decisiones organizacional. (p.79).

Por esta razón, el pensamiento positivo se reconoce como un elemento imprescindible para el bienestar, hacia el éxito en la realización personal del individuo, pues induce a las personas a vivir su propio presente, con mayor conciencia y sentido de responsabilidad. De la misma manera, consigue enfocar al hombre en la esencia misma de lo realmente importante para sí mismo, haciéndole vislumbrar el futuro con una mayor dimensión y perspectiva. Amaya (2020), explica que:

Las organizaciones están pasando por una transformación fundamental en todo el mundo, dejando de lado la idea de las organizaciones tradicionales, rígidas, requiriéndose de organizaciones participativas, más planas, con menos niveles jerárquicos, donde se produzca mayor acercamiento de todos lo que la integran, con una participación más activa de todo el equipo. (p.79).

Así lo afirma Varona (2010), quien igualmente plantea la necesidad de la creación de imágenes positivas, de donde es posible generar un rico ambiente, narrativo de cómo puede ser el futuro y cómo pudiera guiar la actividad actual de las organizaciones. De manera que, estos pensamientos positivos generan automáticamente paz, relajación, alegría al interior de cada individuo, haciéndole sentir una mejor persona. En tal sentido, el pensamiento positivo puede considerarse, más que un estilo de vida, una técnica auto promovida legítima, para reprimir la depresión y acrecentar la autoestima, como una figura capaz de mejorar el bienestar psíquico-físico de la persona. Con referencia a ello, autores como Gutiérrez et al., (2020), apuntan:

Debe tenerse en cuenta que las políticas de empleo son afectadas por el entorno político y la dinámica económica-social, éstas enrumban los destinos de la nación y el comportamiento de la población. Cada política diseñada debe estar bien pensada para el beneficio de la sociedad y aislada de los intereses políticos de un partido. La fuerza de trabajo en Venezuela puede encauzarse a un proceso de desarrollo de adentro hacia afuera, retomando las antiguas teorías de especialización de 


\section{Revista científica, arbitrada e indizada, bajo la modalidad electrónica.}

competencias, diversificación productiva y ruptura de la dependencia de la renta petrolera. (p.40).

De igual forma, en el contexto laboral, el pensamiento positivo como elemento orgánico genera aproximaciones diversas, lo cual le erige como factor clave en el desarrollo de procesos con lugar en el contexto organizacional del sector público, buscando con ello que el trabajador se muestre más motivado, aprovechando las circunstancias, disposiciones y realidades propias de los organismos judiciales, o bien desde la perspectiva teórico práctica, como discierne el autor mencionado, procurando así una gestión operativa más eficiente, eficaz y efectiva, para finalmente alcanzar los resultados esperados. Sumado a ese tenor, debe tomarse en cuenta que tanto el clima físico como laboral, influyen en la sensación de realización propia de cada asociado.

Por ello, se toma en cuenta lo que autores como Buitrago y Henríquez (2019, pág. 83), quienes explican: "En la contemporaneidad las organizaciones occidentales han desarrollado formas de gestión y crecimiento que apuntan al logro de un clima laboral, no solo estable sino agradable". Entre tanto, Vera et al., (2019, pág. 50), refieren que: "La fuerza humana de trabajo es un componente primordial para lograr cambios neurálgicos en organizaciones contemporáneas, hacia una adaptación progresiva conforme a las nuevas tecnologías de la información y comunicación".

En ese sentido, quien asuma el rol de líder, no puede prescindir de esquemas ideáticos positivos, muy por el contrario estos se convierten en condición indispensable, para desarrollar con éxito su misión e integrar a todos los trabajadores a la misma. Para Buitrago (2019, pág. 02a), sucede que: "Resulta necesario para el líder o directivo aprovechar los recursos existentes; con los que pueda llevar su gestión de forma inteligente, humana y aggiornada de cara a los escenarios humanistas que trajo consigo la contemporaneidad, globalización, desarrollo social/ pluripolar/ multicéntrico".

De tal manera, se infiere que una organización liderada bajo un enfoque optimista es más eficaz, pues al estar impregnada con pensamientos positivos, se estimula en ella la identificación, inspiración, motivación, confianza y creatividad en la búsqueda de soluciones, incrementando la conciencia de las oportunidades y por ende, superando más 


\section{Revista científica, arbitrada e indizada, bajo la modalidad electrónica.}

rápido y con éxito las adversidades que afronten. En opinión de Buitrago et al., (2020), sucede que:

Ante el vertiginoso contexto contemporáneo que tuvo origen con la globalización, al interior de las organizaciones públicas surgen fenómenos socialmente notables como el poder y su ejercicio, el cual se ha convertido en vértice del imperio para los entes u órganos del sector publico hispanoamericano, inmersos en una tormenta de intereses $y$ propuestas yuxtapuestas, que han generado un clima de competitividad absoluta, donde quien persiga su prolongación, es exhortado al sincretismo comunicacional del presente. (p.84).

Conforme a lo anterior, es así como el fenómeno "satisfacción laboral" adquiere una especial relevancia, el cual según Robbins (2011), corresponde al estado emocional que deriva de pensamientos positivos o placenteros, con ocasión a la percepción subjetiva de las experiencias laborales del trabajador, más específicamente: de si el cargo que ocupa le compensa o permite robustecer los valores personales que este tenga. Contrariamente, la insatisfacción laboral, constituye la respuesta emocional negativa -pesimismo- hacia el cargo, traduciéndose en patrones de comportamiento evasivos, irritables, irascibles, egoístas, perniciosos, erráticos, llegando incluso a ignorar o negar los valores propios: corrompiéndose. Buitrago (2019), afirma:

El apego a las estructuras clásicas de gestión gerencial en las organizaciones, estimula la perversión a la sumisión, corrompiendo el desarrollo de procesos que deberían ser plásticos, translucidos e incluso luminosos, generando así fenómenos como el denominado organizaciones perversas. Todo líder necesita seguidores, camina adelante, guía. (p.05b).

Al advertir los planteamientos anteriores, puede inferirse la utopía que representa pensar en funcionarios públicos felices, quienes voluntariamente decidan realizar sus actividades bajo parámetros de orgullo y entusiasmo. Sin embargo, tal manera de pensar no reviste su imposibilidad, mucho menos si las organizaciones públicas comprenden los grandes beneficios que ello desencadenaría, con ocasión al sentido de lealtad y compromiso institucional adquirido por los trabajadores, contrario a cuando se presenta o experimenta 


\section{Revista científica, arbitrada e indizada, bajo la modalidad electrónica.}

insatisfacción con su trabajo, llegando a pensar que no ejercerían la función pública a tal nivel, de no ser por el beneficio económico incorporado.

Con base a las ideas anteriores, ante tal escenario embargado por el pesimismo, la psicología positiva resulta necesaria, como lo afirman Cuadra et al., (2010), para contrarrestar una serie de prácticas, que no por ser comunes dejan de ser dañinas e improductivas, toda vez que resulta ilógico pensar que trabajadores insatisfechos, desmoralizados y desanimados pudieran dar lo mejor de sí en su ejercicio laboral.

Así entonces, la ausencia de entusiasmo, vehemencia y entrega tiene su origen en la carencia de inspiración y motivación en el individuo, con relación a sus actividades personales y laborales, por lo cual cabe la pregunta: ¿Que trabajador que no esté motivado con su vida laboral, puede sentirse satisfecho?. Sin motivación en los trabajadores, muy difícilmente se logra una organización exitosa. Así, por el contrario, el entusiasmo que se genera en su ejercicio laboral, como elemento del pensamiento positivo, genera un sello indeleble en el resultado exitoso.

Por otra parte, cabe destacar que durante el desarrollo de la investigación original, de donde se desprende el presente manuscrito, se desarrolló un proceso hermenéutico con cual pudo reconocerse que la constante generación de novedosos, plurales y diversos conocimientos que trajo consigo la era de la información, los cuales intiman a las organizaciones públicas a reestructurar tanto los principios, como los preceptos que la rigen, hacia formas básicas o evolutivas que les constituya como organizaciones pensantes; sobre lo cual Buitrago (2019, pág. 05b), refiere con ello, dejan atrás el paradigma de organizaciones perversas. Con relación a lo descrito en este párrafo, autores como Araujo (2020), explican:

La hermenéutica (...), se define en sus diferentes escuelas por su interés de interpretar, en principio, textos de tipos documentales escritos, para precisar su verdadero significado, esto es, mostrar de forma clara y más allá de toda duda razonable el marco del sistema de representación epistemológica, espaciotemporal, ideológicas y siempre condicionado por su carga histórica, que intenta comunicar algo, no solo sobre el ámbito temático o problemático parte del proceso de observación documental 


\section{Revista científica, arbitrada e indizada, bajo la modalidad electrónica.}

encaminado al diálogo intertextual y contextual, simboliza por sí mismo un ejercicio hermenéutico. (p.15).

En este orden de ideas, el optimismo ha sido estudiado ampliamente por los psicólogos, bajo diferentes acepciones: el optimismo disposicional propuesto por Carver \& Seller en 1981, la esperanza defendida por Zinder durante el año 2000 y el estilo explicativo descrito por Peterson \& Seligman en el año 1984. Conforme a los planteamientos antes descritos, se propone al optimismo como consecuencia al pensamiento positivo, asociado a un mejor estado de ánimo, mayor satisfacción con la vida, mejor estado de salud psíquica y física, el éxito académico, laboral y deportivo; buena salud; además una existencia más próspera.

Asimismo, a los fines de erigir bases sostenibles e inteligentes, la gerencia debe corresponderse proporcionalmente con la complejidad humana, en el entendido que la misma comprende un conjunto de hombres y mujeres, seres profundos, ávidos de líneas de acción, en términos físicos y psicológicos, así como una atención integral, que les empodere a la consecución de logros personales cohesionados de forma armónica con los organizacionales.

En tal sentido, con relación al sector público venezolano, pudiera decirse que tal y como lo refieren Caruso \& Salovey (2009), los gerentes deben ser capaces tanto de aprovechar las oportunidades como de lidiar con las perturbaciones. Así pues, hoy en día es fundamental que los líderes aprendan a apropiarse de sus emociones, y saber contener con inteligencia, asertividad y respeto las de sus colaboradores, procurando ello se convierta en una oportunidad, para mejorar la interacción y la productividad de todos los miembros del equipo.

Por otro lado, aunque las emociones han adquirido gran importancia para los nuevos modelos de gestión organizacional, algunos directivos y gerentes no cuentan con cualidades, ni habilidades para apropiarse de las mismas. Lograr ser un líder emocionalmente inteligente, configura formas de comportamiento equilibrado, con apropiación de sus emociones y sentimientos, con la capacidad para afrontar cualquier 


\section{Revista científica, arbitrada e indizada, bajo la modalidad electrónica.}

tribulación, al tomar en cuenta la huella emocional y comprender emociones colectivas, difusas e individuales.

Conforme a lo anterior, se lograría con ello una comunicación organizacional efectiva, al orientar el pensamiento y las acciones al alcance de los objetivos propuestos, pues cuando el líder logra moderar las señales y emociones que trasciende, asegura su entendimiento e incluso comprensión, lo cual es ideal para el bienestar de la organización. En ese sentido, corresponde a los líderes gerenciar la vida laboral psicocompetitivamente, así como generar y robustecer en su entorno una salud tanto física como mental sostenible, proponiendo nuevos paradigmas, proyectando un estilo de comportamiento positivo, competitivo y proactivo que haga factible actitudes y conductas asertivas, tanto en el trabajo como en su dinámica social y personal.

Según Soto \& Pelekais (2011), con el advenimiento de diversos fenómenos sociales que cuentan su presencia en el ámbito organizacional, surge la necesidad de gerenciar la vida laboral, ya que en toda actividad humana compleja se encuentra presente de forma constante el conflicto; lo cual es una constante en las relaciones interpersonales. Así mismo, los centros de trabajo no escapan de ello, es un hecho inevitable que se presenta a diario, generado por diferencias en ideas, valores y creencias de cada individuo que conforma al entramado orgánico.

Continúan las citadas autoras explicando, que los conflictos, han sido valorados de formas distintas así como contradictorias; para algunos es la fuente del desarrollo, para otros crea desorden, caos. Por lo tanto, constituye un obstáculo al desarrollo de las instituciones y a la productividad en el trabajo. Entre tanto, las organizaciones públicas venezolanas, echan en falta líderes con competencias para hacer frente a los retos existentes y típicos de la realidad social contemporánea.

Igualmente, resulta necesario dejar claro que en la contemporaneidad se destacan y por mucho...aquellos líderes capaces de darle sostenibilidad y posicionamiento a los organismos, entes e instituciones aliadas a dicho sector, que ofrezcan valor agregado en su entorno e inspiren a quienes trabajan con ellos; tomando en cuenta siempre que la 


\section{Revista científica, arbitrada e indizada, bajo la modalidad electrónica.}

incompatibilidad de intereses, valores, propósitos, metas aunado a la escasez de recursos, dan oportunidad al desarrollo de conflictos.

Del mismo modo, se debe identificar en las organizaciones la carencia de estima, reconocimiento y respeto así como también los factores que originan todo ello, lo cual puede generar conflictos con ocasión a emociones negativas como la frustración, desagrado, miedo, tristeza, rabia, pesimismo, desesperanza; por ende, una insatisfactoria vida laboral. Con base a lo planteado, se observa que en la realidad organizacional actual, se requiere de un alto sentido de responsabilidad, lo que merece asumir los retos y desafíos, inherentes a los altos niveles de fluctuación, oscuridad y volatilidad en los escenarios organizacionales, que pudiesen incidir en la manera de gestionar los espacios en los que hace vida el trabajador. En opinión de Proto y Capera (2019), sucede:

Resulta fundamental reflexionar sobre aspectos como la dominación masculina, la eficiencia del poder, la relación política y religión, el sentido práctico de la teoría y la especificidad de los distintos campos (culturales, económico, político, intelectual y social). Estos confluyen en la dinámica de las sociedades modernas inmersas en el sistema capitalista que establece nuevos temas al interior de la agenda que constituyen los intereses de una ciencia en particular teniendo como referencia los intereses de los grupos que representan hegemónicos encargados de enunciar un tipo de ciencia en función de intereses subjetivos y no de procesos interculturales que sean coherentes con la democratización del saber y la diversidad de pensamientos para la sociología política de Nuestra América. (p.61).

En ese orden de ideas, se requiere empoderar a los trabajadores en general a la consecución de metas personales y organizacionales. Por tal razón, los líderes deben saber generar tanto energía como entusiasmo en otros, inspirándoles para crear un entorno positivo, productivo, efectivo y frugal. Adicionalmente, es prudente precisar que el éxito no es producto del azar, no es cuestión de suerte; sino de acción, teniendo su arraigo en una matriz de pensamiento estratégico; los verdaderos líderes tienden a considerarse personas ganadoras, optimistas, esperanzadoras, perseverantes, con las suficientes potencialidades racionales y emocionales como para afrontar cualquier escenario que la vida le plantee. 


\section{Revista científica, arbitrada e indizada, bajo la modalidad electrónica.}

Así entonces, de allí que el propósito del líder sea alcanzar la gerencia de vida laboral, enfrentando las adversidades con inteligencia, logrando así así trabajadores más felices y productivos. No obstante, la calidad de vida laboral depende en gran medida de las acciones ejecutadas por la organización. Finalmente, debe asumirse que cuanto mayor conocimiento se tenga, más efectivas serán sus acciones; no obstante, es preciso tener una mayor destreza reflexiva basada en el pensamiento positivo, generando políticas organizacionales más ajustadas a la realidad organizacional en la cual se esté inmerso.

\section{MARCO TEÓRICO}

Pensamiento positivo: eslabón hacia una gerencia de vida laboral efectiva a favor de la fuerza humana de trabajo en organismos públicos Venezolanos

En el desarrollo de la investigación original de la cual se desprende el artículo en cuestión, se logró concretar un cuerpo escrito, que condensó las teorías más significativas con relación al pensamiento positivo y la gerencia de vida laboral. En ese sentido, al redactar el extenso en cuestión, se exponen extractos de la misma, para de algún modo, quizás más prácticos, ofrecer los planteamientos que diferentes autores generaron en cada una de sus doctrinas. Sin embargo, en este segmento, se dará cuenta de algunos postulados, que en opinión de quienes colaboraron en la realización de este escrito, representaron mayor aporte.

Del mismo modo, es preciso destacar que el pensamiento positivo, así como también la gerencia de vida laboral, configuran fenómenos de naturaleza humana, por tanto, pueden y de hecho son abordados por expertos en ciencias humanas, sociales, políticas, administrativas, económicas y tantas otras, como las militares por ejemplo, que exponen su interés por abordar desde un enfoque científico el comportamiento humano y organizacional, de más está decir, que el pensamiento positivo, representa a su vez, el contexto intangible donde las ideas encuentran su oportunidad más álgida. Para Toscano (2020), sucede que:

La gestión de personal en las organizaciones demanda importantes retos para los directivos de diversas empresas en el mundo, debido principalmente a las transformaciones que han ocurrido en las últimas 


\section{Revista científica, arbitrada e indizada, bajo la modalidad electrónica.}

cuatro décadas en distintos ámbitos con respecto a las dinámicas sociales, en general, y a la estructuración y gestión de las organizaciones, en particular. (p.129).

Conforme a lo anterior, y en el desarrollo de la investigación matriz de este manuscrito, surgieron diferentes interrogantes a saber: ¿En qué consiste el pensamiento positivo? - ¿Cuál es el alcance del pensamiento positivo? - ¿De qué forma el pensamiento positivo favorece los procesos humanos? - ¿Cuáles son los procesos que se ven favorecidos con el pensamiento positivo? - ¿De qué manera el pensamiento positivo puede convertirse en estrategia organizacional? - ¿Que necesidades, aspiraciones e intereses pueden satisfacerse con pensamiento positivo?; así también otras tantas, por lo que en opinión de los autores, no habrían suficientes hojas en el mundo para lograr formularlas todas en su máxima expresión. En opinión de Peña et al., (2020), sucede que:

El talento es un recurso escaso y valioso; por ello, desarrollarlo debe ser una prioridad en la agenda estratégica de cada una de las organizaciones interesadas en trascender $y$ ser exitosa. Algunas organizaciones lo ven como una barrera, mientras que otras lo conciben como una ventaja; este aspecto se podrá convertir en una ventaja competitiva cuando la organización trabaje en políticas y programas enfocados al óptimo desarrollo de habilidades y capacidades de sus colaboradores. Estas acciones influyen positivamente para solucionar los problemas de escasez y la guerra de talento. (p.04).

Por ello, surgen escritos como este, quizás con el interés personal e investigativo de sus autores, por comprender de que va el pensamiento positivo; contextualizado en organismos del sector publico venezolano, como alguna forma de auscultar, cuales son los esquemas de pensamiento que acuden a la mente del funcionario público con ejercicio profesional en Venezuela, a los fines de esclarecer su propósito, como actor clave en el desarrollo de procesos con lugar en ese sector, tan demandante como vapuleado en los últimos años, por otras instituciones, y por qué no decirlo...criticado en diferentes países y otros miembros de la comunidad internacional.

Así pues, cabe indicar que el pensamiento positivo reviste un proceso neurálgico; donde la creatividad, inventiva, empatía e inteligencia social se cohesionan en el raciocinio 


\section{Revista científica, arbitrada e indizada, bajo la modalidad electrónica.}

humano, para otorgar a cada escenario, situación, persona e incluso perspectivas, un marco favorecedor; donde a partir de un halo de optimismo, se visualiza casi todo acontecimiento de la mejor forma posible, lo cual incluso pudiera hacer las veces de terapia neuronal, como una bocanada de aire fresco a la sistematización de ideas en la mente de cualquiera que así lo eche en falta.

Entre tanto, para autores como Varona (2010), el pensamiento positivo configura la estructura mental y afectiva de la persona, se puede desarrollar con la práctica de técnicas apropiadas, lo cual exige disciplina y tiempo para lograrlo. El mismo, permite la exteriorización del individuo, lo separa de la preocupación centrada en sí mismo, enfoca su interés en los aspectos positivos de su entorno, del mundo, incrementa los sentimientos de solidaridad, pudiendo actuar de manera más altruista e incluso filantrópica.

Igualmente, el pensamiento positivo ha sido asociado con la capacidad creativa para solucionar problemas, resolver situaciones conflictivas, de manera más efectiva, con el optimismo junto a la capacidad de aprender, y en particular con la agudeza de percibir y entender las cosas positivas. En materia de pensamiento, igualmente destacan las expertas en la temática Soto \& Pelekais (2011), que la comunicación interpersonal resulta fundamental para el intercambio de valores, principios, intereses, compromisos, en pro de la organización, su funcionamiento y prolongación, para así concretar un alto desempeño y éxito administrativo.

En ese sentido, la comunicación efectiva, configura el vínculo que permite una gestión comprometida; el estilo de pensamiento proactivo que a la vez impulsa dicho proceso comunicacional y la psicocompetitividad en líderes potenciales; todo lo cual está orientado a la consecución de procesos y cambios exitosos. Destacan las autoras citadas en el párrafo anterior Soto \& Pelekais (2011), que corresponde a los líderes asumir nuevos paradigmas, basados en un estilo de pensamiento positivo, a través del cual se observen sus competencias, promoviendo comportamientos asertivos en el contexto donde se desenvuelvan.

Así entonces, se da cuenta del contenido que representa el pensamiento positivo como fenómeno y por qué no, proceso neurálgico, en el desarrollo de las diferentes 


\section{Revista científica, arbitrada e indizada, bajo la modalidad electrónica.}

circunstancias que cada trabajador pudiera afrontar, teniendo en cuenta que si bien, el extenso se desprende de una investigación cualitativa, que en su momento abordara al pensamiento positivo, lo hizo desde un enfoque administrativo, como apoyo al desarrollo de nuevas aristas, que edifiquen las estructuras cognoscitivas inherentes a la estrategia gerencial contemporánea y por ende al contexto laboral y administrativo.

Por otro lado, la gerencia de vida laboral configura un proceso mucho más complejo, que el que ocupara el interés de los autores en los párrafos anteriores, pues en ella se conjugan un conglomerado de fenómenos que no sólo la enriquecen, sino que también la diversifican, otorgándole un carácter multifacético, el cual pudiera ser aprovechado por disciplinas y ciencias más allá de las administrativas; teniendo en cuenta que en la misma encuentran oportunidad: la inteligencia ética colectiva, inteligencia social, cultura organizacional, filosofía orgánica, liderazgo, comunicación, prospectiva organizacional, logística inversa, toma de decisiones e incluso cuestiones tan prácticas y absolutamente tangibles como ornamento, iluminación, espacio físico, entre otros factores de índole diversa. Pérez \& Zurita (2016), refieren que:

Es válido rescatar que en el estudio de este fenómeno, existen dos perspectivas que por sí mismas abordan dimensiones diversas del mismo, en ello se cuentan la Calidad de Vida del Entorno Laboral y la Calidad de Vida Laboral Psicológica. La primera tiene como propósito suministrar una mejor calidad de vida a sus funcionarios mediante la consecución de objetivos organizacionales, enfocados en las condiciones de trabajo y en los elementos estructurales. Por su parte, la segunda perspectiva busca estudiar los factores asociados a la organización y a las relaciones interpersonales que determinan la experiencia del funcionario sobre su nivel de satisfacción, bienestar y salud. (p.173).

En ese sentido, cabe preguntarse: ¿Por qué desarrollar un constructo teórico sobre gerencia de vida laboral?. Como se ha hecho notar en el discurso del presente extenso, los autores persiguen comprender de qué forma esta pudiera ser favorecida o aún más potencializada por el pensamiento positivo, para con ocasión a ello, observar si en la gestión pública venezolana se echan en falta fenómenos como estos o si por el contrario se tiene mucho de los mismos. Es menester dejar claro, que ambas categorías exhiben naturaleza diversa; sin embargo su base científica se instruye y apoya en las ciencias sociales y en consecuencia comprenden elementos con carácter humanista. En ese sentido Lumbreras et al., (2020), explican que: 


\section{Revista científica, arbitrada e indizada, bajo la modalidad electrónica.}

Los resultados de los estudios sociológicos que hemos desarrollado, destacan que las organizaciones aliadas al sector público en general, manifiestan una gran necesidad por adoptar metodologías y herramientas que mejoren la gestión directiva, con el fin de garantizar la eficiencia y calidad de los servicios públicos en mayor medida, extendiendo así su alcance y satisfacción de necesidades, Se requiere en estos escenarios mejorar el desempeño de las organizaciones públicas, lo cual implica tomar acciones encaminadas a la gestión del talento humano, atendiendo los resultados de la investigación empírica para mejorar la percepción de CVL de los profesionales y funcionarios públicos, realizar evaluaciones integrales del desempeño periódicas y fomentar la gestión directiva para el logro de los objetivos organizacionales. (p.94).

Para Fuendzalia citado por Mattei (2012), la gerencia de vida laboral, muestra mayor interés por el trabajador, desarrollando un estudio de los elementos de trabajo en las que participa directamente el individuo. Esta señala aspectos subjetivos de la vida laboral, concediendo a los trabajadores un papel destacado, con relación a las de condiciones físicas de trabajo, entre las que destacan: mobiliario, tecnología, clima físico, higiene, limpieza, sanidad y seguridad, así como otros elementos mucho más neurálgicos a nivel personal, que tienen que ver con el salario, compensación, motivación, satisfacción, oportunidades de ascenso, desarrollo y participación en el diseño de cargos a ejercer, así como otros tantos estímulos sociales, económicos, laborales, administrativos e incluso psíquicos.

Por otra parte, Lau \& May (1998), refieren a la calidad de vida laboral como el seriado de condiciones ambientales de trabajo favorables, que protegen y promueven la satisfacción de los trabajadores mediante recompensas, seguridad laboral y oportunidad de desarrollo personal. Por su parte Chiavenato (2009), reconoce la calidad de vida en el trabajo como el alcance con el que los miembros de la organización pueden satisfacer las necesidades, interés y aspiraciones de los trabajadores que ejerzan la actividad organizacional.

En opinión de Fernández (2005), la dependencia que se crea entre la institución y el trabajador representa un elemento determinante, además de decisivo de la forma y calidad de vida de este último, que abarca no sólo su aspecto físico, sino también psicológico, además de emocional, esto tanto en la jornada laboral como fuera de esta. Igualmente, de 


\section{Revista científica, arbitrada e indizada, bajo la modalidad electrónica.}

una manera indirecta, afectará también a la vida de aquellas personas con las que el trabajador se relacione en entornos sociales, deportivos, familiares, románticos, entre otros.

Es por ello, que el líder psicocompetitivo, debe reconocer al hombre en sí mismo, luego desde su experiencia como portadores y hacedores primeramente de cultura. Al gerenciar el conocimiento desde como aprender, desaprender o deconstruir y reconstruir conocimiento en sí mismo y hacia su equipo de trabajo. Cabe destacar en este apartado, que al hablar de gerencia de vida laboral, se alude a la gestión efectiva de la misma, hacia el alcance real de lo que el trabajador pudiera reconocer como calidad de vida laboral.

Asimismo, desde el punto de vista de Méndez (2006), se refiere al aspecto cualitativo del modo de vida, expresado en libertad social y condiciones para que los individuos puedan alcanzar su desarrollo, es decir, la definición se centra en las necesidades relativas al bienestar. En concreto, la calidad de vida laboral, configura un fenómeno psicosocial, cuyo enfoque pude ser humanista, ético, bioético, gerencial, psicométrico, político, jurídico, médico, tecnológico, estadístico e incluso cultural.

A la luz de los planeamientos anteriores, pudiera decirse que la gerencia de vida laboral tiene mucho que ofrecer como fenómeno sí, pero también como el contexto donde los procesos humanos, culturales, sociales, administrativos, tecnológicos, y de disímil naturaleza tienen lugar, en ella se reúnen y cohesionan elementos tangibles e intangibles, por lo tanto valdría la pena abordarle no sólo desde un enfoque académico, sino también estratégico, practico, operacional, y vale decirlo; científico, pues en ella se generan diferentes escenarios donde los expertos pueden identificar elementos nuevos que hasta entonces no hayan sido percibidos.

Indistintamente, el abordaje científico a la gerencia de vida laboral representa un reto académico para los estudiosos en ciencias administrativas, pues la misma al tener una naturaleza de carácter diverso, obliga al investigador a explorarla desde diferentes perspectivas científicas: humanas, sociales, culturales, políticas, económicas, entre otras, lo cual genera cierta complejidad en el desarrollo de procesos indagativos; como el que diera origen a la investigación de donde emerge este extenso. 


\section{Revista científica, arbitrada e indizada, bajo la modalidad electrónica.}

En ese sentido, la calidad de vida laboral reviste un conjunto de principios, los cuales apuntan al factor humano como el elemento más importante en la organización. En ello, se plantea que los trabajadores son dignos de confianza, responsables tanto como capaces de generar contribuciones valiosas, por lo que deberían ser tratados con dignidad y respeto. Finalmente, Zohurul \& Siengthai (2009), plantean que la calidad de vida laboral, reviste una condición favorable para los trabajadores, la cual puede ser gestionada a través del cuidado de la salud, al igual que las actitudes positivas de los directivos hacia ellos; favoreciendo la mejor disposición de ser y hacer en el talento humano hacia un desempeño más efectivo.

\section{METODOLOGÍA}

\section{Diseño}

El presente artículo, es producto de una investigación científica terminada, ajustada al paradigma cualitativo, con apoyo al método etnográfico, y cuyo enfoque fuera introspectivo-vivencial, intitulada: "Pensamiento positivo para potenciar la gerencia de vida laboral en organismos públicos"; en la cual se aplicaron como técnicas: el registro de grabación, la observación participante, la entrevista semiestructurada y la técnica documental como herramientas durante el proceso indagativo.

Igualmente, además de todo lo descrito en el párrafo anterior, debe hacerse especial mención que los hallazgos logrados con esta investigación, están mayormente integrados por el contenido informativo generado tras el desarrollo y completación de las entrevistas, realizadas a los sujetos seleccionados para tal fin, con el propósito de plantear laudos epistémicos, apoyados en la abstracción e interpretación de los datos recogidos durante todo el procedimiento indagativo, así como la auscultación de los fenómenos abordados. Ello permitió el desarrollo coherente de un abordaje epistemológico, representado en un paradigma, su enfoque y la respectiva corriente del pensamiento adoptada durante el proceso.

Según con lo establecido por Rodríguez et al., (2009), la investigación de la cual se desprende el artículo en cuestión, dispuso en su desarrollo de una diversidad de 


\section{Revista científica, arbitrada e indizada, bajo la modalidad electrónica.}

paradigmas, métodos y estrategias que constituyeron el pluralismo fortalecedor de este tipo de trabajo. En el caso del presente estudio, se seleccionó el enfoque interpretativo, el cual se centró en el estudio de los significados de las acciones humanas y de la vida social. En opinión de Valles (1997), esta perspectiva intenta sustituir las nociones científicas de explicación, predicción y control del paradigma positivista por los conceptos de comprensión, significado y acción, según lo establece.

Conforme a lo anterior, el artículo en cuestión expone extractos del contenido axiológico, epistemológico, ontológico del constructo logrado tras la terminación de la investigación referida, e incluso parte de los hallazgos alcanzados por la misma. Condensando elementos doctrinales, investigativos, epistémicos logrados por el proceso investigativo y analítico de bases doctrinales, así también a las propuestas teóricas diversos autores, consecuentes a trabajos investigativos ya completados y seleccionados para tal fin, así como también la información, datos recolectados en las distintas entrevistas realizadas, generando así una masa informativa y documental que diera cuenta del recorrido investigativo llevado a cabo durante el proceso investigativo.

En tal sentido, con la terminación de la investigación de la cual se desprende este escrito, se logró generar laudos epistémicos e ideográficos; de forma dinámica, múltiple y holística, cuestionando la existencia de una realidad externa y valiosa. El paradigma cualitativo seleccionado para el desarrollo de la indagación, se centró en el estudio de la realidad organizacional, con lugar en algunas fiscalías adscritas al Ministerio Público en Venezuela, a los fines de comprender los significados que las personas implicadas, otorgaron a los elementos que la integran, para estudiar sus creencias, intenciones, motivaciones y otras características del proceso de gestión, no observables directamente, ni susceptibles de experimentación.

Igualmente, el diseño de la investigación intitulada: "Pensamiento positivo para potenciar la gerencia de vida laboral en organismos públicos" y de donde se origina este artículo, estuvo dividido en cuatro momentos, donde cada uno configuró un propósito diferente: El Momento I, donde se recogió la información concerniente a la situación abordada, desde la óptica del investigador. De la misma manera se formularon los 


\section{Revista científica, arbitrada e indizada, bajo la modalidad electrónica.}

propósitos y las interrogantes de la investigación, las implicaciones, así como la delimitación del estudio.

Por otro lado, el Momento II, donde se condensaron los referentes epistémicometodológicos de la investigación, marcando el camino a seguir para alcanzar los propósitos descritos en el Momento I, lo cual implicó identificar una teoría de conocimiento con la debida antelación, que permitiera establecer la realidad investigada, referida al análisis del pensamiento positivo como habilidad potenciadora de la gerencia de vida laboral en organismos públicos del Estado Zulia.

Del mismo modo, el Momento III, permitió se analizaran aspectos teóricos relativos a la temática considerada en la investigación, a fin de sustentar bajo referentes pertinentes al estudio en cuestión. Persiguiendo a través de un proceso reflexivo, la identificación de las categorías preliminares que perfilaron el mismo. De allí que se desarrollara la teorización de entrada, con apoyo a las bases teóricas referidas a Pensamiento Positivo y Gerencia de Vida Laboral.

\section{Participantes}

Esta investigación fue desarrollada abordando la realidad organizacional de tres (03) oficinas adscritas al Ministerio Público en Venezuela, el cual es uno de los órganos del Poder Ciudadano, encargado de ejercer la acción penal en nombre del Estado, único e indivisible y actualmente está bajo la dirección y responsabilidad de la actual Fiscalía General de la República. Ahora bien, se conformó una muestra integrada por sesenta y tres (63) sujetos, funcionarios públicos que para el tiempo en que se desarrollara la misma ejercían sus cargos de forma libre, sostenida y continuada sin ningún tipo de limitación.

Conforme a lo anterior, y luego de un proceso de observación consecuente se redujo a un muestreo intencional de tres (03) sujetos, adscritos a las unidades de análisis seleccionadas para tal fin y quienes formaron parte del ciclo de entrevistas. De acuerdo con Taylor \& Bogdan (2010), los investigadores cualitativos, definen típicamente su muestra, sobre una base que puede evolucionar a medida que el estudio progresa. De allí que la decisión sobre el mejor modo de obtener los datos y de quién o quiénes obtenerlos se toman en el campo, ya que es la propia información obtenida la que va guiando al muestreo. 


\section{Revista científica, arbitrada e indizada, bajo la modalidad electrónica.}

Igualmente, cabe acotar de forma especial, que al mismo tiempo se hizo la selección previa de tres (03) informantes clave, funcionarios del Ministerio Público, quienes conocían la temática referida al Pensamiento positivo y Gerencia de vida laboral en las organizaciones seleccionadas; con los que se tuvo contacto desde los inicios indagativos in situ, y de quienes se recibió un gran cumulo informativo de interés medular para el desarrollo y completación del trabajo que diera origen al presente artículo.

\section{Instrumentos}

Para la elaboración de esta investigación, fueron seleccionadas como técnicas la observación participante, la entrevista semiestructurada y además la técnica documental, con la cual muy especialmente se logró aprovechar el mayor cúmulo de contenidos obtenidos de documentos escritos: públicos y privados, en físico e incluso digital: Artículos científicos, Tesis de investigación científica, así como otros datos relativos al marco legislativo, estatutario, jurisprudencial que fuera necesario revisar ante la naturaleza de los organismos públicos de las unidades de análisis abordadas durante el proceso investigativo.

Igualmente, fueron consideradas técnicas como el registro de grabación, la cual resultó de gran valía para el la recopilación y tratamiento del mayor cúmulo de información, esto permitió condensar una mayor masa de contenido, generada por parte de los trabajadores cumpliendo su rol como sujetos de investigación durante las entrevistas realizadas un situ. Así también, se hizo uso de los instrumentos indicados para el aprovechamiento de las diferentes técnicas aplicadas, en la recolección y registro digital de la información, como lo fueron la grabadora digital, la computadora, el diario de campo y la matriz de análisis de la categoría.

\section{Procedimientos}

Esta investigación, se realizó en un periodo que fue desde Septiembre de 2014, hasta Enero del 2020, ocupando 64 meses para su completación. Resulta ineludible mencionar que el registro de grabación, fue la técnica utilizada desde el primer, hasta el último momento relativo a la recolección de datos in situ. De acuerdo con Martínez (2009), en tal 


\section{Revista científica, arbitrada e indizada, bajo la modalidad electrónica.}

procedimiento prevalece el carácter subjetivo tanto en el análisis como en la interpretación de resultados.

Así también, fue aplicada la técnica de observación participante, la cual en opinión de Urrego (2009), permite al investigador recoger por si mismo información relacionada con el fenómeno abordado, favoreciendo su identificación, reconocimiento, abordaje y profundización desde el lugar del observador, apreciando con la mayor inteligencia e imparcialidad posible.

Por otro lado, para la selección, discriminación, decantación, análisis e interpretación de contenidos, se utilizó la técnica documental, de la cual Finol \& Nava (2004), plantean que en ella, el investigador examina los materiales a través de dos (02) tipos de lectura: un examen preliminar de los elementos de presentación y de referencia, para determinar la existencia de datos importantes; una posterior realizada en forma analítica para determinar el significado, el valor de cada documento.

Al mismo tiempo, con el propósito de fundar bases teóricas que cimentaran el apoyo a la doctrina original, propuesta por la investigación original de la cual se desprende el artículo en cuestión, se utilizó una matriz de análisis de la categoría, la cual fue empleada como guía para condensar la información de tipo documental y a todo dato generado en las unidades de análisis seleccionadas. De acuerdo con Hurtado (2008), esta constituye un registro organizado de información, para proceder a extraer conclusiones científicas que generen un nuevo conocimiento.

Ahora bien, la entrevista semiestructurada fue la aplicada a los funcionarios públicos, seleccionados como sujetos de estudio, la cual según Finol \& Camacho (2006), presenta un margen más o menos grande de libertad, para formular las preguntas y el entrevistado es libre de dar sus propias respuestas, sin sentirse constreñido por la naturaleza de la pregunta. Cabe acotar, que previamente a la celebración de cada entrevista, donde fueran aprovechados la grabadora digital y el diario de campo como instrumentos investigativos. 


\section{Revista científica, arbitrada e indizada, bajo la modalidad electrónica.}

Igualmente, el registro completo de la información y datos recolectados, tanto en la indagación documental, como en la recogida conjunta durante la realización de las entrevistas, se logró el almacenaje de los escritos donde se apoya este trabajo, lo cual se concretó con el empleo del registro sistemático en computadora, utilizando una carpeta digital diferente para cada unidad de análisis abordada, para posteriormente ser procesado, como de hecho ocurrió finalmente y de donde se generaron disímiles documentos, tal es el caso de este artículo.

Así pues, la información condensada durante todo el proceso indagativo, fue sometida al análisis coherente e interpretación profunda, desde un enfoque lógico, reflexivo y dialéctico, que como lo expresan Hurtado \& Toro (2007), permite desarrollar la capacidad creadora y de cuestionamiento propia del ser humano. Para ello, se tomó en cuenta no solo la realidad social que circundó el periodo en el cual fuera llevada a cabo la investigación, también se advirtió la realidad interna en el contexto abordado, unidades de análisis seleccionadas: oficinas fiscales, adscritas al Ministerio Público.

De igual manera, fue empleada la técnica del análisis de contenido, la cual según Ander (2009), integra diversos recursos que permiten abordar los eventos de estudio, hechos, situaciones, textos, autores, video, cine, con el interés de profundizar en su comprensión. Finalmente, en este estudio, para el análisis e interpretación de los hallazgos, se hizo la triangulación teórica. Según Finol \& Camacho (2006), esta supone la compilación de diversos datos como métodos, que aplicados a un evento, hecho o situación problema, generan la comprensión e interpretación de estos, desde la perspectiva individual y combinada de los procedimientos utilizados.

\section{RESULTADOS}

Desde la óptica jurídica y conociendo el marco constitucional, legal y normativo que impera en Venezuela, así como de la realidad social que se refleja de forma neurálgica en los escenarios organizacionales, además de las funciones propias de la fiscalía del Ministerio Público, es posible ofrecer laudos epistémicos al contexto laboral en este organismo, visto como contexto de interés investigativo para las ciencias administrativas, el cual aún al distinguirse favorablemente de otras instituciones de la administración pública y 


\section{Revista científica, arbitrada e indizada, bajo la modalidad electrónica.}

organismos autónomos, no se ha logrado ajustar de manera efectiva a una óptima gestión gerencial, donde tanto la actitud de los funcionarios como la calidad en los procesos con lugar en su interior sean contundentemente efectivos.

No obstante, hay que reconocer los grandes esfuerzos invertidos en esa temática, además de lo que respecta a los logros en materia de beneficios salariales, las herramientas tecnológicas con que se cuenta, la disposición de equipos, materiales e instrumentos para ejercer la función pública, así como también espacios físicos adecuados. De allí que en esta investigación, se logró observar con mayor precisión, de forma exhaustiva el desarrollo de las dinámicas laborales en las que un grupo de funcionarios de diversos niveles tanto profesionales como económicos participaron.

Conforme a ello, es posible generar lineamientos gerenciales del pensamiento positivo y su capacidad de potenciar la gerencia de vida laboral en los funcionarios públicos, tomando en cuenta las entrevistas a diferentes sujetos involucrados en la investigación y la observación directa de todos los factores intervinientes en tal proceso. Ahora bien, desde la óptica investigativa, durante el proceso indagativo se percibieron actitudes poco entusiastas de los funcionarios auscultados, pesimismo en los resultados a ser logrados, desconfianza en su estructura emocional, así como falta de responsabilidad en la entrega oportuna de su trabajo.

Por otro lado, fue posible apreciar deficiencias en las tareas ejecutadas por los funcionarios públicos, que aplicaron como sujetos de estudio, específicamente en las acciones laborales cotidianas, propias de la función pública, ejercida en el ámbito judicial, caracterizadas por el desinterés, la apatía, negligencia, a razón de estar caracterizada por una jornada laboral sin horario establecido, que consecuencialmente provee poco o nada de tiempo libre, limitando así el esparcimiento y el desarrollo de una vida social y familiar plena, que hoy en día se constituyen en clamores primordiales del bienestar físico, de las relaciones interpersonales positivas, del crecimiento personal y la felicidad.

Como corolario de lo anterior, es necesario precisar que los fiscales titulares y auxiliares adscritos al Ministerio Público Venezolano, están sujetos integralmente a su dinámica laboral, ejemplo de ello es que deben mantener sus dispositivos telefónicos 


\section{Revista científica, arbitrada e indizada, bajo la modalidad electrónica.}

activos las 24 horas del día, igualmente presentan una restricción en la salida de la entidad territorial en la que laboran, aún en días feriados y fines de semana. En el mismo tenor, se recogen de comentarios diversos, durante diferentes encuentros investigativos tanto con fiscales, como con otros funcionarios adscritos al Ministerio Público, que en su entorno laboral se genera un cúmulo de ideas e inclinaciones tanto negativas como prejuiciosas.

A la luz de las aseveraciones anteriores, y en lo que respecta a los sujetos entrevistados, las realidades del trabajo con las que tienen que lidiar fiscales titulares, auxiliares y otros colaboradores en las oficinas adscritas al Ministerio Público Venezolano, influyen en el comportamiento del funcionario, generando en su accionar patrones de comportamiento autolimitado, errático, inhibiendo con ello el desarrollo de relaciones interpersonales satisfactorias con compañeros y colegas, generando así episodios de frustración, ansiedad, apatía, tristeza y desapego al igual que una escasa interacción con intervinientes del mismo contexto, en aras de evitar señalamientos indeseables que pudieran disgregar su reputación.

Conforme a ello, lo antes narrado, se adminicula a la falta de confianza en la institución a integrantes de la misma y la visión sesgada en lo relativo a su indemnidad o privilegio y libertad de crear, contrayendo la innovación, sesgando su propia iniciativa, posiblemente como consecuencia de tratarse de una organización pública de estructura vertical jerárquica. Así entonces, al relacionar la problemática planteada, profundizando en el pensamiento positivo, para potenciar la gerencia de vida laboral en los funcionarios públicos, logra apreciarse tanto dificultades como carencias dentro del Ministerio Público Venezolano, al observar la matriz ideática de los funcionarios, inclinada hacia actitudes y sentimientos incongruentes con la efectiva ejecución de tareas, actividades y funciones propias de la administración pública.

\section{CONCLUSIONES}

En este estudio se analizaron los hallazgos logrados con base en las entrevistas realizadas a tres (03) fiscales titulares, funcionarios adscritos al Ministerio Público, como contexto abordado en el proceso investigativo; realizándose, con posterioridad, la categorización, triangulación, análisis e interpretación de los mismos, que posteriormente 


\section{Revista científica, arbitrada e indizada, bajo la modalidad electrónica.}

fueron confrontados con las opiniones y planteamientos de los autores consultados en las referencias teóricas. De allí que, en este apartado se procede a la emisión de conclusiones con atención a los propósitos establecidos desde el inicio.

En primer término, las diligencias iniciales durante el proceso investigativo se avocaron a identificar los elementos del pensamiento positivo, presentes en los funcionarios adscritos a los Organismos Públicos del Estado Zulia, seleccionados para tal fin. Se observó que el personal adscrito a las tres (03) fiscalías auscultadas, presentó falencias en cuanto a su responsabilidad operativa, haciéndose necesario aplicar estrategias de concienciación en la función pública, con la finalidad de aumentar sus niveles de rendimiento.

De la misma manera, al entrevistar a los tres (03) sujetos de investigación, seleccionados para consumar la serie de entrevistas, se observó en estos, algunas debilidades con ocasión a las inadecuadas condiciones de trabajo, dando lugar a la falta de estabilidad en el desempeño, lo que imposibilitó la efectividad de los trabajadores en ejercicio de la función pública, dando oportunidad a la ineficacia que se traduce en el incumplimiento de los objetivos estatales.

Ahora bien, en segundo término, se propuso el desarrollo de acciones orientadas a generar los beneficios ofrecidos por el pensamiento positivo, a favor de los funcionarios adscritos a los organismos públicos seleccionados como el contexto en el que se llevase a cabo este estudio, específicamente en oficinas fiscales, adscritas al Ministerio Público con sede en el Estado Zulia, Venezuela. Con lo cual se pudo plantear que la matriz de pensamiento positivo, deriva en escenarios de bienestar, interés personal, energía positiva en las relaciones interpersonales y el desarrollo de la capacidad de innovación en dicho contexto.

Conforme a lo anteriormente planteado, cabe referir que lo contrario conduce a las organizaciones públicas donde el pensamiento caótico, confuso, incoherente se hace presente y multiplica, cuestión que limita el desenvolvimiento cabal a los funcionarios públicos, esto último exige esquemas de reforma en la matriz estructural de la organización, tanto en ejercicio del poder público, como el aprovechamiento de una experiencia laboral 


\section{Revista científica, arbitrada e indizada, bajo la modalidad electrónica.}

que hasta entonces cada trabajador haya alcanzado, dando ocasión a transformaciones en el comportamiento de quienes ejerzan cargos públicos.

Igualmente, con relación a la caracterización de los elementos que conforman la gerencia de la vida laboral, resulta necesario expresar que en los organismos públicos auscultados, se encontraron vacíos con relación a la operatividad de los procedimientos efectuados en tales oficinas fiscales, específicamente en la resolución de los casos que en estas se procesan, pues los funcionarios no cuentan con las herramientas estratégicas indicadas, dando ello lugar a un desempeño operativo inaceptable, lo cual acarrea consecuencias de gran magnitud, que no solo juegan en contra del organismo en cuestión, sino también vulnera los intereses de ciudadanos que esperan se haga justicia, bien como actores (procesados) o como victimas sin que puedan recibir una respuesta efectiva.

Asimismo, se observó que en las entidades públicas seleccionadas como unidades de análisis, se trabaja de manera aislada, es decir, no se labora en equipo, sin planes concretos de operatividad, dificultando los procesos, y más aún, entorpeciendo con ello el alcance de los resultados que la colectividad espera, desencadenando así el incumplimiento de los objetivos estatales de la gestión pública, al no tomar decisiones indicadas y efectivas que correspondan, en el momento oportuno.

En tal sentido, se observaron patrones de estrés, agotamiento por el exceso de trabajo, insatisfacción laboral, descontrol en las emociones y sentimientos, conflictos entre los trabajadores, entre éstos y los ciudadanos como usuarios del servicio, además de tropiezos en los procesos relativos a la comunicación, dificultades en la transmisión de información, ausencia de valores en la gestión de trabajo, así como también otros factores disruptivos: retrasos en su ejecución operativa, impuntualidades en el horario de trabajo, desánimo, negligencia, todo lo cual impide las decisiones adecuadas, oportunas, violentando entonces los fines estatales para las cuales fueron creadas tales oficinas públicas.

\section{REFERENCIAS BIBLIOGRÁFICAS}

Amaya, D. (2020). Clima organizacional y sus tipos en instituciones de salud pública de Colombia. Consensus (Santiago) - Revista Interdisciplinaria De Investigación, 4(1), 


\section{Revista científica, arbitrada e indizada, bajo la modalidad electrónica.}

78

90. Recuperado

a partir

de

http://pragmatika.cl/review/index.php/consensus/article/view/55

Araujo, J. (2020). Socialismo del siglo XXI: degeneración de la democracia venezolana. SUMMA. Revista disciplinaria en ciencias económicas y sociales, 2(1), 13-40.

Ander, E. (2009). Métodos y Técnicas de Investigación Social. Técnicas para la recogida de datos e información. Volumen IV. Buenos Aires. Argentina. Lumen Humanitas.

Buitrago, R., Hernández, M. \& Hernández, P. (2020). Teoría del eterno retorno: Hontanar en los modelos de gestión organizacional contemporánea. SUMMA. Revista Disciplinaria En Ciencias económicas Y Sociales, 1(2), 82-103. Recuperado a partir de https://aunarcali.edu.co/revistas/index.php/RDCES/article/view/92

Buitrago, R. (2019a). Filosofía orgánica: una aproximación crítica desde la perspectiva del hombre venezolano contemporáneo. Cuadernos Latinoamericanos de $\begin{array}{llllll}\text { Administración, } & 15(29), & 1 & - & 14 . & \text { Doi: }\end{array}$ http://www.redalyc.org/articulo.oa?id=409661113002

Buitrago, R. (2019b). Liderazgo reformador: una perspectiva cualitativa desde la plasticidad de la industria farmacéutica. Consensus (Santiago) - Revista Interdiscipliaria De Investigación, 3(2), 3-40. Recuperado a partir de http://pragmatika.cl/review/index.php/consensus/article/view/34

Buitrago, R., Henríquez, C. (2019). Humor y Optimismo al Interior de las Organizaciones Públicas Venezolanas hacia una Gerencia de Bienestar. Revista Latitude, (12), 82 104.

Caruso, D. \& Salovey, P. (2009). El gerente emocionalmente inteligente. Chile. Editorial Jossey Bass.

Cuadra, A., Veloso, C., Mora, Y., Reyes, L., Vilca, J. (2010). Efecto de un programa de psicología positiva e inteligencia emocional sobre la satisfacción laboral y vital. Revista Salud y Sociedad. 1(2). 101-112. Doi.: http://www.saludysociedad.cl/index.php/main/article/view/17/37

Chiavenato, I. (2009) Gestión del talento humano. México. Ediciones Mc Graw Hill.

Fernández, R. (2005). Administración de la responsabilidad social corporativa. España. International Thomson Editores.

Finol, M., Camacho, H. (2006). El proceso de investigación científica. Venezuela. Editorial Ediluz.

Finol, T., Nava, H. (2004). Procesos y Productos en la Investigación documental. Venezuela. Editorial Ediluz.

Granados, I. (2011). Calidad de vida laboral: historia, dimensiones y beneficios. Revista IIPSI, 14(2), $271-276$.

Gutiérrez, J., Romero, J., Balestrini, L. (2020). Algunas consideraciones sobre la fuerza de trabajo en Venezuela. SUMMA. Revista Disciplinaria En Ciencias económicas Y Sociales, 1(2), 28-43. Recuperado a partir de https://aunarcali.edu.co/revistas/index.php/RDCES/article/view/88

Hurtado, I \& Toro, J. (2001). Paradigmas y Métodos de Investigación en tiempos de cambio. Venezuela. Editorial Episteme.

Revista Saperes Universitas

ISSN 2642-4789

Vol. 3 No. 2 


\section{Revista científica, arbitrada e indizada, bajo la modalidad electrónica.}

Hurtado, J. (2008). Metodología de la Investigación Holística. Venezuela. Ediciones de la Fundación Servicios y Proyecciones para América Latina (SYPAL).

Lau, R., May, B. (1998). A Win- Win Paradigm for Quality of Work life and Bussiness Performance. Human Resource Developpment Quarterly. 9(3). 211- 227.

Lumbreras, M., Hernández, I., Méndez, P., Dosamantes, L., Cervantes, D., García, A., Cortez, H. (2020). Influencia de la calidad de vida laboral sobre el desempeño de trabajadores de hospitales públicos de México: una visión desde la gestión directiva. salud publica mex., 62(1):87-95.

Martínez, M. (2009). La investigación cualitativa etnográfica en educación. Manual teórico-práctico. México. Editorial Trillas.

Mattei, A. (2012). Coaching y calidad de vida laboral en los Empleados del Sector Petrolero. (Tesis de Postgrado no publicada). Universidad Privada Rafael Belloso Chacín. Venezuela.

Méndez, C. (2006). Clima organizacional en Colombia. El IMCOC: Un método de análisis para su intervención. Colombia. Centro Editorial Universidad del Rosario.

Peña, P., Vargas, A. \& Rodríguez, J. (2020). Administración de la gestión humana: un caso de las empresas privadas de Florencia-Caquetá. Revista Estrategia Organizacional, 9(1). https://doi.org/10.22490/25392786.3647

Pérez, D. \& Zurita, R. (2016). Calidad de vida laboral en trabajadores de salud pública en Chile. Salud \& Sociedad, 5(2), $\quad 172-180$. https://doi.org/10.22199/S07187475.2014.0002.00002

Proto, F., Capera, J. (2019). Pierre Bourdieu y sus aportes a la sociología política contemporánea. SUMMA. Revista disciplinaria en ciencias económicas y sociales, $1(2), 44-63$.

Robbins, S. (2011). Comportamiento organizacional. México. Editorial Mc Graw Hill.

Rodríguez, G., Gil, J. y García, E. (2009). Metodología de la investigación cualitativa. España. Editorial Algive.

Soto, M., Pelekais, C. (2011). Gerencia de vida laboral: Estilos de pensamiento y psicocompetitividad. Formación gerencial y transformación de líderes. España. Editorial Académica Española.

Valles, M. (1997). Técnicas cualitativas de investigación social. Reflexión metodológica y práctica profesional. España. Editorial Síntesis.

Vera, J., Buitrago, R., Rosales, N., Hernández, M. ( 2019). Del Ideático Boricua Contemporáneo: Desmitificando al Gamer desde las Nuevas Realidades del Trabajo. Revista Latitude, (12), 49 - 69.

Varona, M. (2010). El circulo de la comunicación. Serie: Comunicación empresarial. España. Editorial Netbiblio.

Taylor, S. \& Bogdan, R. (2010). Introducción a los métodos cualitativos. E.E.U.U. Editorial Book Print 


\section{Revista científica, arbitrada e indizada, bajo la modalidad electrónica.}

Toscano, C., Vesga, J. \& Avendaño, B. (2020). Calidad de vida en el trabajo y su relación con el engagement. Acta Colombiana de Psicología, 23(1), 128-146. https://doi.org/10.14718/ACP.2020.23.1.7

Urrego, A. (2009). La investigación acción participativa en el contexto socio educativo: estrategia metodológica en la producción del conocimiento para la acción social. Revista Educare. 13(2). 154-166.

Zohurul, I. \& Siengthai, S. (2009). Quality of Work Life and Organizational Performance: Empirical Evidence from Dhaka Export Processing Zone. This paper is prepared for the ILO Conference on Regulating for Decent Work, to be held at the International Labour Office. Suiza. 